\title{
PARADA CARDÍACA: PRINCIPAIS COMPLICAÇÕES OCASIONADAS PELA SÍNDROME DA RABDOMIÓLISE
}

\author{
HEART STOP: MAIN COMPLICATIONS CAUSED BY RABDOMIÓLISE \\ SYNDROME
}

\author{
Amauri dos Santos Araujo ${ }^{1}$ \\ Izadora Nunes da Silva ${ }^{2}$ \\ Glaucia dos Santos Silva ${ }^{3}$ \\ Vanessa Maria do Nascimento ${ }^{4}$
}

\begin{abstract}
RESUMO: A parada cardiorrespiratória (PCR) é uma situação clínica que acarreta prioridade de atendimento devido à necessidade de intervenções rápidas e eficazes. OBJETIVO: identificação da produção científica a nível internacional e nacional sobre as principais complicações fisiopatológicas advindas da síndrome rabdomiólise, MÉTODOS: trata-se de um estudo de revisão integrativa, realizada na bases de dados Biblioteca Virtual em Saúde (BVS), da Literatura Latino-Americana e do Caribe em Ciências da Saúde (LILACS), Base de Dados em Enfermagem (BDENF), Scientific Eletronic Library Online (SciELO) e Medical Literature Analysisand Retrieval System Online (MEDLINE). RESULTADOS E DISCUSSÃO: O presente estudo possibilitou compreender e identificar a definição da condição patológica e sua ampla vertente e etiologia. Entretanto, além da sua relevância, identificou-se a limitação de conhecimento pelos profissionais da saúde, oriundo da escassez de estudo publicado na área. CONCLUSÃO: A falta de conhecimento pode causar algumas situações que interferem na atuação de toda a equipe na assistência prestada. É conferido ao enfermeiro a responsabilidade do suprimento do carrinho de parada e todos os materiais necessários, bem como os cuidados dispensados durante todo o evento.
\end{abstract}

Palavras chave: Lesão muscular. Parada Cardíaca. Rabdomiólise.

\footnotetext{
${ }^{1}$ Enfermeiro. Mestrando em enfermagem pelo PPGENF/EENF/UFAL. Professor da Faculdade de Tecnologia de Alagoas. E-mail: amauriaraujo.sms@gmail.com.

2 Graduanda em Enfermagem pela Faculdade de Tecnologia de Alagoas.

${ }^{3}$ Graduanda em Enfermagem pela Faculdade de Tecnologia de Alagoas.

${ }^{4}$ Graduanda em Enfermagem pela Faculdade de Tecnologia de Alagoas.
} 
ABSTRACT: Cardiopulmonary arrest (CRP) is a clinical situation that leads to priority care due to the need for rapid and effective interventions. OBJECTIVE: To identify international and national scientific production on the main pathophysiological complications arising from rhabdomyolysis syndrome. METHOD: This is an integrative review study, conducted in the Virtual Health Library (VHL), Latin American and Caribbean Health Sciences (LILACS), Nursing Database (BDENF), Scientific Electronic Library Online (SciELO) and Medical Literature Analysisand Retrieval System Online (MEDLINE). RESULTS AND DISCUSSION: The present study made it possible to understand and identify the definition of the pathological condition and its broad aspect and etiology. However, besides its relevance, it was identified the limitation of knowledge by health professionals, arising from the scarcity of studies published in the area. CONCLUSION: Lack of knowledge can cause some situations that interfere with the performance of all staff in the assistance provided. The nurse is given the responsibility of supplying the stopping cart and all necessary materials, as well as the care given throughout the event.

Keywords: Muscle Injury. Cardiac Arrest. Rhabdomyolysis. 


\section{INTRODUÇÃO}

A Síndrome do Esmagamento ou rabdomiólise decorre de uma lesão do tipo esmagamento em grandes massas musculares, comumente envolvendo coxa ou panturrilha. Caracteriza-se por dor, fraqueza muscular e urina acastanhada, sendo associada à Insuficiência Renal Aguda (IRA) (PHTLS, 2011; HERLON, 2010). Define-se como uma entidade comum, podendo conter etiologia multifatorial, como trauma ou injúria direta (choque e queimaduras), excesso de atividade muscular, etc. (CABRAL 2012; ARTHUR, 2011).

A concentração de potássio em níveis elevados pode resultar em arritmias cardíacas, podendo ser fatais em alguns casos de altas concentrações. Além disso, a mioglobina livre aparece na urina com coloração de chá ou refrigerante à base de cola podendo causar danos (PHTLS, 2011). Atualmente, as causas são subdivididas em: trauma ou injúria direta (choque e queimaduras); Excesso de atividade muscular; Defeitos enzimáticos hereditários: como doença de Mc Ardle (HERLON, 2010).

Em uma célula de atividade normal, sem estar sujeita a um estresse fisiológico, a membrana celular se encontra íntegra e que a partir do momento que ocorre uma lesão, a permeabilidade da membrana aumenta e a enzima creatina quinase (CK) passa para o plasma sanguíneo, aumentando sua concentração, com isso, suas manifestações clínicas vão além do local do trauma (CABRAL, 2012; ARTHUR, 2011).

Em consequência do dano, a membrana da célula relacionada à sua concentração entre o espaço intra e extracelular é mantida pela bomba iônica sódiopotássio-ATPase, que transporta o potássio para dentro e o sódio para fora das células (CABRAL, 2012). A destruição do músculo libera uma molécula de mioglobina e há liberação de potássio em alta concentração, essa concentração em níveis elevados pode resultar em arritmias cardíacas, podendo ser fatais já que pode provocar parada cardíaca. 
A gravidade desta condição está relacionada à magnitude, duração da força compressiva e dimensão do músculo afetado. Os sinais e sintomas resultantes da lesão muscular por esmagamento não são limitados ao local do trauma, ocorrendo manifestações sistêmicas. Portanto, a avaliação do indivíduo com rabdominiólise é importante para evitar maiores complicações intervindo de forma imediata em urgências (MENDES, 2014; COSTA, 2013).

Já a mioglobina que é liberada extravasa para o sangue aumentando a formação de cilindros tubulares, prejudiciais a filtração glomerular, favorecendo a IRA, como consequências danosas (MENDES, 2014; CABRAL, 2012; ARTHUR, 2011). No entanto pensar nas complicações e condutas a serem realizadas, emerge a seguinte questão norteadora para o presente estudo: "Quais as principais complicações cardíacas que acomete ao paciente com a síndrome de rabdomiólise?". Logo, o objetivo desse estudo, além de responder a pergunta norteadora compete estudar a caracterização dos tipos de lesões ocasionados pela síndrome, identificação das consequências cardíacas advindas do esmagamento e avaliação a qualidade das publicações sobre a referida temática.

\section{MATERIAL E MÉTODO}

Trata-se de um estudo de revisão integrativa, no qual é permitida a síntese de múltiplos estudos publicados, possibilitando conclusões gerais a respeito de uma determinada área de estudo. Desta feita, as pesquisas são analisadas fornecendo subsídios para tomadas de decisões, como também auxiliando na síntese do conhecimento sobre determinado assunto, apontando inclusive possíveis preenchimentos de lacunas encontradas na literatura estudada (WHITTEMORE; KNAFL, 2005; SILVA et al., 2012; STUM; SANTOS; RESSEL, 2012).

Para a construção da revisão integrativa é preciso percorrer seis etapas distintas: a identificação do tema e seleção da hipótese; o estabelecimento de critérios para inclusão e exclusão de estudos/amostras ou busca na literatura; a definição das informações a serem extraídas dos estudos selecionados; avaliar os 
estudos incluídos na revisão integrativa; a discussão dos resultados permeia-se a partir da interpretação e síntese dos resultados; e, a apresentação da revisão deve ser clara e completa para permitir ao leitor avaliar criticamente os resultados (WHITTEMORE; KNAFL, 2005; ARAUJO; MOTA, 2014; SOUZA; SILVA; CARVALHO, 2010).

Além disso, a revisão integrativa inclui a análise de pesquisas relevantes que dão suporte para a tomada de decisão e a melhoria da prática clínica, possibilitando a síntese do estado do conhecimento de um determinado assunto, além de apontar lacunas do conhecimento que precisam ser preenchidas com a realização de novos estudos (MENDES et al, 2008).

A coleta de dados foi realizada nos meses de novembro de 2018 à janeiro de 2019, nas bases de dados da Biblioteca Virtual em Saúde (BVS), da Literatura Latino-Americana e do Caribe em Ciências da Saúde (LILACS), Base de Dados em Enfermagem (BDENF), Scientific Eletronic Library Online (SciELO) e Medical Literature Analysisand Retrieval System Online (MEDLINE). Foram utilizadas estratégias de busca a partir dos Descritores de Ciências da Saúde (DeCS) "Rabdomiólise", "Parada Cardíaca" e "Lesão muscular", por meio do formulário próprio das bases de dados, com as combinações do booleano "AND", harmonizando conforme as etapas de cada base de dados: "Rabdomiólise" AND "Parada Cardíaca" AND "Lesão muscular".

Como critérios de inclusão foram considerados o ano de publicação entre 2011 a 2016, e que estejam publicados em português, inglês ou espanhol, no qual o mesmo deve encontrar- se disponível em formato de texto completo e gratuito. Como critérios de exclusão, monografias e teses; e aqueles que não responderam à pergunta norteadora e aqueles que não atendem aos critérios de inclusão estabelecidos.

Sob esses critérios foram encontrados 22 artigos. Destes artigos 11 foram selecionados para análise que devem ser lidos minuciosamente, destacando os que atendem aos objetivos deste trabalho afim de melhor organizar e tabular os dados. 


\section{RESULTADOS E DISCUSSÃO}

Dos 11 artigos localizados, foram selecionados 05 para composição final da amostra - por retratarem o objetivo da pesquisa, sendo lidos e tabulados conforme título da pesquisa, base de indexação, ano de publicação, objetivo, tipo de estudo e síntese do estudo, de acordo Quadro 1.

Quadro 1 - Artigos selecionados para a realização da revisão integrativa. Maceió AL, 2019.

\begin{tabular}{|c|c|c|c|c|c|}
\hline $\begin{array}{l}\text { Título da } \\
\text { Publicação }\end{array}$ & $\begin{array}{c}\text { Base de } \\
\text { Indexação/ } \\
\text { natureza do } \\
\text { trabalho }\end{array}$ & $\begin{array}{c}\text { Ano de } \\
\text { Publicaçãol } \\
\text { local de } \\
\text { publicação }\end{array}$ & $\begin{array}{l}\text { Objetivos do } \\
\text { Trabalho }\end{array}$ & $\begin{array}{l}\text { Tipo de } \\
\text { Estudo }\end{array}$ & $\begin{array}{c}\text { Síntese do Estudo } \\
\text { Relacionada à Questão } \\
\text { Norteadora }\end{array}$ \\
\hline \begin{tabular}{|} 
Rabdomiólise, \\
insuficiência \\
renal aguda e \\
parada cardíaca \\
secundária ao \\
status distônico \\
em uma criança \\
com acidúria \\
glutárica tipo I \\
(GA1)
\end{tabular} & $\begin{array}{c}\text { Periódico } \\
\text { CAPES com } \\
\text { acesso CAF } \\
\text { /Artigo } \\
\text { científico }\end{array}$ & $\begin{array}{l}\text { 2012/ } \\
\text { Elsevier }\end{array}$ & \begin{tabular}{|c|} 
Ressaltar a \\
importância de que \\
pacientes com GA1 \\
e estado distônico \\
podem estar em \\
risco de \\
rabdomiólise com \\
risco de vida agudo, \\
insuficiência renal e \\
lesão neurológica \\
em qualquer \\
momento da vida. \\
\end{tabular} & $\begin{array}{c}\text { Relato de } \\
\text { caso }\end{array}$ & $\begin{array}{l}\text { Caso clínico de um } \\
\text { menino de } 8 \text { anos de } \\
\text { idade com acidúria } \\
\text { glutárica tipo I que } \\
\text { apresentou rabdomiólise, } \\
\text { insuficiência renal aguda } \\
\text { e parada cardíaca } \\
\text { secundária ao status } \\
\text { distônico em associação } \\
\text { com doença febril. }\end{array}$ \\
\hline $\begin{array}{c}\text { Rabdomiólise } \\
\text { após hipotermia } \\
\text { terapêutica após } \\
\text { parada cardíaca } \\
\text { traumática }\end{array}$ & $\begin{array}{l}\text { Periódico } \\
\text { CAPES com } \\
\text { acesso CAF } \\
\text { /Artigo } \\
\text { científico }\end{array}$ & 2011/Elsev & $\begin{array}{c}\text { Descrever a } \\
\text { rabdomiólise como } \\
\text { uma complicação } \\
\text { rara da terapêutica } \\
\text { a hipotermia, onde o } \\
\text { seu reaquecimento } \\
\text { podem interromper } \\
\text { sua progressão. }\end{array}$ & $\begin{array}{c}\text { Relato de } \\
\text { caso }\end{array}$ & $\begin{array}{l}\text { Um homem de } 32 \text { anos } \\
\text { de idade esteve } \\
\text { envolvido em um } \\
\text { acidente de moto e } \\
\text { sofreu uma parada } \\
\text { cardíaca pós- traumática } \\
\text { anóxica (pTCA): } \\
\text { assistolia seguida de } \\
\text { fibrilação ventricular. }\end{array}$ \\
\hline
\end{tabular}




\begin{tabular}{|c|c|c|c|c|c|}
\hline $\begin{array}{c}\text { Síndrome } \\
\text { Maligna dos } \\
\text { Neurolépticos: } \\
\text { Complicações, } \\
\text { Desfechos e } \\
\text { Mortalidade }\end{array}$ & \begin{tabular}{|c|} 
Periódico \\
CAPES com \\
acesso CAF \\
/Artigo \\
científico
\end{tabular} & 2016/Springer & $\begin{array}{c}\text { Fornecer } \\
\text { informações atuais } \\
\text { sobre as } \\
\text { complicações da } \\
\text { SMN e relatar os } \\
\text { preditores de } \\
\text { mortalidade. }\end{array}$ & $\begin{array}{l}\text { Análises } \\
\text { estatísti } \\
\text { cas }\end{array}$ & $\begin{array}{c}\text { Relata que A } \\
\text { complicação mais } \\
\text { prevalente foi a } \\
\text { rabdomiólise }(30,1 \%) \text {. } \\
\text { Outras complicações } \\
\text { comuns foram } \\
\text { insuficiência respiratória } \\
\text { aguda }(16,1 \%), \text { lesão } \\
\text { renal aguda }(17,7 \%), \\
\text { sepse }(6,2 \%) \text { e outras } \\
\text { infecções sistêmicas. A } \\
\text { taxa de mortalidade não } \\
\text { ajustada foi de } 5,6 \% \text {. }\end{array}$ \\
\hline $\begin{array}{c}\text { Morte cardíaca } \\
\text { súbita recorrente } \\
\text { abortada com } \\
\text { convulsões e } \\
\text { rabdomiólise } \\
\text { devido à } \\
\text { hipocalemia } \\
\text { induzida pela } \\
\text { bulimia: relato de } \\
\text { um caso. }\end{array}$ & $\begin{array}{c}\text { Periódico } \\
\text { CAPES com } \\
\text { acesso CAF } \\
\text { /Artigo } \\
\text { científico }\end{array}$ & $\begin{array}{c}\text { 2014/Med } \\
\text { Chile }\end{array}$ & $\begin{array}{c}\text { Mostrar que a } \\
\text { bulimia e o abuso de } \\
\text { furosemida ou } \\
\text { laxantes podem } \\
\text { causar Morte } \\
\text { cardíaca súbita com } \\
\text { convulsões } \\
\text { sintomáticas e } \\
\text { rabdomiólise. }\end{array}$ & $\begin{array}{l}\text { Relato de } \\
\text { caso }\end{array}$ & $\begin{array}{l}\text { Relata que uma mulher } \\
\text { de } 25 \text { anos de idade com } \\
\text { história de bulimia } \\
\text { associada ao uso } \\
\text { abusivo de furosemida e } \\
\text { laxantes desde os } 15 \\
\text { anos de idade, migrânea } \\
\text { desde a puberdade, } \\
\text { abscessos renais aos } 20 \\
\text { anos e rabdomiólise de } \\
\text { causa desconhecida aos } \\
24 \text { anos. }\end{array}$ \\
\hline $\begin{array}{c}\text { Hipocalemia } \\
\text { grave induzida } \\
\text { por furosemida } \\
\text { com } \\
\text { rabdomiólise } \\
\text { sem parada } \\
\text { cardíaca }\end{array}$ & $\mid \begin{array}{c}\text { Periódico } \\
\text { CAPES com } \\
\text { acesso CAF } \\
\text { /Artigo } \\
\text { científico }\end{array}$ & $\begin{array}{l}\text { 2013/BMCWo } \\
\text { men's Health }\end{array}$ & \begin{tabular}{|c|} 
Afirmar que a \\
hipocalemia \\
induzida por abuso \\
de diuréticos é uma \\
emergência com \\
risco de vida.
\end{tabular} & $\begin{array}{l}\text { Relato de } \\
\text { caso }\end{array}$ & $\begin{array}{l}\text { Hipocalemia induzida por } \\
\text { furosemida extrema com } \\
\text { um nível sérico de } \\
\text { potássio de } 1,1 \mathrm{mmol} / \mathrm{I} \\
\text { é rara e está associada a } \\
\text { parada cardíaca com } \\
\text { risco de vida e } \\
\text { rabdomiólise. }\end{array}$ \\
\hline
\end{tabular}

FONTE: elaborado pelos autores, 2019. (Dados da pesquisa).

De acordo com os estudos de Jamuar, et al. (2012), a Rabdomiólise e lesão renal aguda associada a distonia e espasmo muscular têm sido bem descritos na literatura. No entanto, nota-se que há uma escassez de informações sobre pacientes com distúrbios e um distúrbio de movimento secundário aos danos nos gânglios da base apresentando rabdomiólise aguda, onde a causa exata da rabdomiólise não foi determinada.

Distonia grave em pacientes com acidúria glutárica tipo I (GA1) e gânglios da base podem gerar danos, mas não suficientes para o desenvolvimento de rabdomiólise. Os autores ressaltam ainda em seu estudo que as crises hiperpiróxicas são a causa mais provável no estudo de caso abordado, enquanto que supõe-se que níveis aumentados de 3-hidroxiglutarato com inibição resultante 
síntese da ATP mitocondrial no músculo pode ter contribuído para rabdomiólise em seu paciente (JAMUAR et al., 2012).

Reitera-se que os dois pacientes descritos apresentavam histórico de um distúrbio do movimento crônico, mas não está claro se esses pacientes tiveram piora da distonia durante esses episódios de rabdomiólise (JAMUAR et al, 2012). Entretanto, Rosa et al. (2005), defendem que a rabdomiólise possui causas raras e decorrem da incapacidade em produzir a quantidade de ATP adequada às necessidades das células musculares, por deficiência de enzimas do metabolismo dos glícidos, lípidos ou nucleósidos. Normalmente surgem na infância, sob a forma de dor, fraqueza muscular e mioglobinúria recorrentes após exposição a estímulos que em condições normais não condicionam necrose muscular (ex. exercício físico ligeiro, infecções virais ou jejum).

Para Siqueira et al. (2008), quando retratando a farmacologia, rabdomiólise comporta- se como uma complicação relevante em pacientes cuja a dislipidemia está bem estabelecida, juntamente com as enzimas hepáticas e miopatia. A rabdomiólise, que se caracteriza por necrose muscular, mioglobinúria e insuficiência renal aguda.

No geral, as estatinas estão relacionadas a um pequeno risco de miopatia que pode progredir para rabdomiólise fatal ou não fatal. A incidência está relacionada com a dose e o uso concomitante de agentes que compartilham a mesma via metabólica das estatinas. A insuficiência renal aguda é a mais temida complicação da rabdomiólise e a principal causa de óbito. O reconhecimento e tratamento precoce podem prevenir a progressão da rabdomiólise (SIQUEIRA et al., 2008).

Segundo Ciapetti et al (2011), A hipotermia ativa foi suspensa e passiva aquecimento iniciado. Os índices de rabdomiólise diminuíram imediatamente e, 06 horas depois, os valores de potássio e os índices de necrose foram normais. Rabdomiólise apareceu durante apesar normovolemia e ausência de drogas vasoativas ou qualquer outra droga que podem causar rabdomiólise. Na ausência de outros óbvios gatilhos, a rabdomiólise foi presumivelmente causada pela hipotermia.

A rabdomiólise relacionada à hipotermia é descrita apenas em casos de longa exposição acidental ao frio que a rabdomiólise foi desencadeada pela hipotermia terapêutica após hipoperfusão e hipoxia causada por trauma (CIAPETTI et al., 
2011). Em estudo de Modi et al. (2016), dentre as complicações associadas à mortalidade a rabdomiólise foi a mais comum $(30,1 \%)$ e esteve presente mais frequentemente em homens do que em mulheres (34\% vs $25 \%$; $p<0,001$ ).

Segundo Rosa et al. (2005), em seu estudo, aborda dados epidemiológicos: nos EUA são descritos cerca de 26.000 casos anuais de rabdomiólise6. A IRA mioglobinúrica ocorre em cerca de $30 \%$ dos casos de rabdomiólise2. Estima-se que 10\%-15\% das IRA são provocadas por rabdomiólise7. A IRA induzida por pigmentos (hemólise, rabdomiólise traumática e não-traumática) é relativamente rara em idosos ( $\geq 65$ anos), comparativamente a grupos etários mais jovens ( $>17$ e $<65$ anos), representando respectivamente $4 \%$ e $12,8 \%$ das causas de IRA. A taxa de mortalidade global dos doentes com rabdomiólise ronda os $5 \%$ existindo uma maior incidência no sexo masculino, sobretudo o grupo associado ao trauma.

No estudo de Finsterer e töllberger (2014), observa-se que a bulimia e o abuso de furosemida ou laxantes podem causar abortos recorrentes morte cardíaca súbita com convulsões sintomáticas e rabdomiólise. Pacientes com bulimia, e que fazem uso adicionalmente, de laxantes ou furosemida correm um risco particular de morte súbita e rabdomiólise e requerem determinação periódica de eletrólitos, e terapia de vigilância psiquiátricos adequados.

Já no estudo de Ruisz et al. (2013), são abordados as manifestações neurológicas da hipocalemia em que incluem a paralisia e rabdomiólise. A Rabdomiólise em hipocalemia pode permanecer assintomática ou se manifestar clinicamente com dores musculares, cãibras e fraqueza. A razão para rabdomiólise na hipocalemia é assumida como isquemia muscular. Potássio fisiologicamente elevado, por exemplo, devido ao exercício, causa vasodilatação e aumenta assim o fluxo sanguíneo muscular. No caso de hipocalemia, o aumento do potássio sérico é prejudicado resultando em isquemia relativa, cãibras musculares e caso de depleção grave, necrose muscular e rabdomiólise. Outro mecanismo pode ser metabolismo dos miócitos levando a disfunção da membrana, resultando em degradação de células musculares ou membrana vazamento. 


\section{CONCLUSÃO}

A Parada Cardiorrespiratória é uma situação preocupante, motivada por uma alta morbimortalidade, mesmo em casos onde possui boas condições para um atendimento bem- sucedido, uma vez que o tempo expressa a necessidade de ações rápidas e eficiente. Após dados coletados permitiram observar que uma equipe bem preparada e atuação harmônica são primordiais. Não só o enfermeiro deve estar apto mais toda a equipe de enfermagem precisa estar capacitada para a constatação de uma parada e conhecer as manobras de suporte básico de vida.

Observou-se a escassez de estudos voltados para a Rabdomiólise levando a Parada cardíaca, o que dificultou ampliação do objeto desse estudo. Com essa problemática a comparação de estudos de casos ficou prejudicada. No entanto foi possível compreender a definição dessa condição patológica e sua ampla vertente e etiologia. Neste ínterim, um ponto que merece destaque, além da sua relevância, é a limitação de conhecimento pelos profissionais da saúde, oriundo da escassez de estudo publicado na área.

A falta de conhecimento pode causar algumas situações que interferem na atuação de toda a equipe na assistência prestada. É conferido ao enfermeiro a responsabilidade do suprimento do carrinho de parada e todos os materiais necessários, bem como os cuidados dispensados durante todo o evento. $O$ treinamento adequado da equipe de enfermagem, em especial daquela que atua frequentemente com pacientes institucionalizados, é vital para o pronto atendimento em parada. Identificar o conhecimento teórico e prático da sobre essa temática é um requisito importante para o planejamento de um treinamento em serviço. 


\section{REFERÊNCIAS BIBLIOGRÁFICAS}

ARTHUR, C. Guyton. Fisiologia humana / Arthur C. Guyton; tradução Charles Alfred esberard. [reimpr.] - Rio de janeiro: Guanabara koogan, 2011.

BOTTON, B. et al. Relato de caso de rabdomiólise em um praticante de esportes radicais rapel e trekking, uma emergência a ser reconhecida. Arquivos Catarinenses de Medicina. Vol. 40, no. 3, de 2011. Disponível em: < http://www.acm.org.br/revista/pdf/artigos/886.pdf>.

CABRAL, G. G.; et al. Insuficiência Renal Aguda devido à Rabdomiólise. ACTA Biomedica Brasiliensia / Volume 3. $n^{\circ} 2,2012$.

COSTA, R.; et al. Lesão renal aguda e rabdomiólise como apresentação da doença de mcardle. Acta Med Port. 2013 Jul-Aug;26(4):463-466.

CIAPETTI, M. et al. Rhabdomyolysis following therapeutic hypothermia after traumatic cardiac

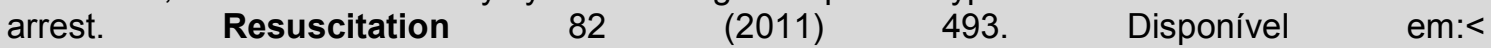
https://www.ncbi.nlm.nih.gov/pubmed/21242021>.

FERREIRA, D. G. Causas, efeitos e tratamento da rabdomiólise. Universidade Castelo Branco e Atualiza - Associação Cultural. 2012.

FINSTERER, J.; TÖLLBERGER, C. Recurrent aborted sudden cardiac death with seizures and rhabdomyolysis due to bulimia-induced hypokalemia. Report of one case. Rev Med Chile 2014; 142: 799-802. Disponível em:< https://www.ncbi.nlm.nih.gov/pubmed/25327327>.

JAMUAR, S. S.; et al. Rhabdomyolysis, acute renal failure, and cardiac arrest secondary to status dystonicus in a child with glutaric aciduria type I. Molecular Genetics and Metabolism 106 (2012) 488-490. Disponível em:< https://www.ncbi.nlm.nih.gov/pubmed/22771013>.

MARTELLI, A.; et al. Aspectos Clínicos e Fisiopatológicos da Rabdomiólise após Esforço Físico Intenso. Persp. Online: biol. \& saúde, Campos dos Goytacazes, 13 (4), 13-22, 2014. Disponível http://ojs3.perspectivasonline.com.br/index.php/biologicas_e_saude/article/view/182>.

MARTINS, H. S.; et al. Emergência Clínicas: abordagem Prática. - 6 ed. ampl. E ver. Barueri, SP: Manole, 2011.MENDES, R.; PEDRO, I; SOUSA, A. R. Síndrome compartimental do antebraço. Rev. Port. Ortop. Traum. 2014, vol.22, n.1, pp. 127-134. Disponível em:< http://www.scielo.mec.pt/scielo.php?script=sci_arttext\&pid=S1646-21222014000100013>.

MODI, S.; et al. Neuroleptic Malignant Syndrome: Complications, Outcomes, and Mortality. Neurocrit Care (2016) 24:97-103. Disponível em:< https://www.ncbi.nlm.nih.gov/pubmed/26223336>.

MONIZ, M. S.; et al. Rabdomiólise como manifestação de uma doença metabólica: relato de caso. Rev Bras Ter Intensiva. 2017; vol.29, n.1, p:111-114.

OLIVEIRA, F. L. Rabdomiólise: revisão bibliográfica com base num caso clinico

de etiologia rara. Clínica Universitária de PediatriA. Faculdade de Medicina da Universidade de Lisboa. Ano lectivo 2015/2016.

PHTLS - Atendimento Pré-Hospitalar ao traumatizado / NAEMT (National Association of Emergency Medical Technícians), Tradução de PHTLS: Prehospital Trauma Life Support - Rio de Janeiro: Elsevier, 2011. 
RABELO, I. B. et al. Rabdomiólise com altos níveis de creatinofosfoquinase, sem evolução para insuficiência renal. Rev Soc Bras Clin Med. 2016 jan-mar; vol.14, n.1, p:38-40. Disponível em:< http://www.sbcm.org.br/ojs3/index.php/rsbcm/article/view/177>.

RUISZ, W.; et al. Furosemide-induced severe hypokalemia with rhabdomyolysis without cardiac arrest. BMC Women's Health. 2013, vol.13, n.30. Disponível em:< https://www.ncbi.nlm.nih.gov/pmc/articles/PMC3710286/>.

WHITTEMORE, R; KNAFL, K. The integrative review: updated methodology. J Adv Nurs. 2005, Dec; vol.52, n.5, p.:546-53. 